\title{
Caractérisations chimiques des ustensiles de cuisine artisanale en aluminium fabriqués au Burkina Faso : cas de Ouagadougou
}

\author{
Jacques SAWADOGO ${ }^{1 *}$, Bernard NISOL ${ }^{2}$, Moussa BOUGOUMA ${ }^{1}$, Tambi RAMDÉ ${ }^{1}$, \\ Guel BOUBIÉ $^{1}$, Claudine BUESS-HERMANN ${ }^{2}$ et Jean Boukari LEGMA ${ }^{1}$ \\ ${ }^{1}$ Laboratoire de Chimie Moléculaire et des Matériaux Equipe Chimie Physique et d'Electrochimie, \\ UFR/SEA, Université de Ouagadougou, 03 BP 7021 Ouagadougou 03, Burkina Faso. \\ ${ }^{2}$ Faculté des Sciences, Services de Chimie Analytique et Chimie des Interfaces (CHANI), \\ Université Libre de Bruxelles, Bruxelles, Belgique. \\ *Auteur de correspondant ; E-mail : jacquischimie@hotmail.fr; genetraore@univ-ouaga.bf \\ Tel. : (+226) $78284066 / 78824246$
}

\section{RÉSUMÉ}

Dans le cadre de la valorisation des matériaux d'origine artisanale du Burkina Faso, deux (02) types d'échantillons d'ustensiles de cuisine (marmites artisanales) en aluminium prélevés dans les différentes zones de la ville de Ouagadougou, ont subi une série d'analyses chimiques. L'artisanat pose le problème de la qualité des produits finis du pays surtout quand on sait qu'ils sont utilisés pour la cuisine de tous les jours. Ainsi, aucun de ces alliages ne devrait être utilisé pour la fabrication d'ustensiles de cuisine, si nous nous référons à la norme française EN 601 en juillet 2004. Ce travail est une contribution à la caractérisation physique et chimique des marmites artisanales au centre du Burkina Faso. L'analyse à la fluorescence X (XRF) indique que la majeure partie des échantillons étudiés, contiennent environ 87,3\% d'aluminium. La diffraction aux rayons X (DRX), la spectrométrie de photoélectrons (XPS), la microscopie optique (MO) montrent que les éléments constitutifs essentiels des échantillons d'ustensiles de cuisine sont: Aluminium, Silicium, cuivre, Magnésium et de Zinc. Enfin, une mesure par colorimétrie permet de mettre en évidence la présence de l'aluminium en solution. (C) 2014 International Formulae Group. All rights reserved.

Mots clés : Alliages d'aluminium, analyses chimiques, DRX, MO, XPS, colorimétrie.

\section{INTRODUCTION}

Au Burkina Faso, les ustensiles de cuisine de fabrication artisanale occupent une place de choix dans l'art culinaire car se prêtant mieux aux conditions de vie des populations les plus vulnérables (Draissia et al., 2004 ; Draissia et al., 2005 ; Ndiaye, 2012). Ces ustensiles sont en général obtenus à partir d'aluminium de récupération de toutes natures (piston, carter, épaves d'avion, canette, etc.) que les fondeurs classent en alliages «durs », « mous » et « normaux » (Ndiaye, 2006; Karbouj, 2008). Les matériaux recyclés, outre l'aluminium, ont une composition chimique diversifiée. Ils contiennent, à l'état de traces, des métaux lourds qui à long terme exposent les populations à des risques réels de 
contamination (Shabestari, 2004 ; Draissia et al., 2005). Compte tenu de l'absence de règles préétablies pour la préparation de la charge de fusion et de la diversité des déchets d'aluminium, chaque alliage est unique. Une évaluation du processus d'élaboration, de l'analyse de couches formées, des propriétés chimiques des ustensiles de cuisine permettront une évaluation de la qualité de ces ustensiles de cuisine. L'objectif principal de cette étude est d'une part de présenter des résultats chimiques et la caractérisation minéralogique de ces ustensiles de cuisine en divers milieux culinaires, avec les objectifs spécifiques pour l'avenir de prévenir les dangers éventuels et d'autre part évaluer leurs résistances à la cuisson par des méthodes électrochimiques.

\section{MATÉRIEL ET MÉTHODES}

\section{Matériel et préparations des échantillons}

Les deux (02) échantillons d'alliages d'aluminium coulés en forme de rondelle ont été prélevés dans deux ateliers de la ville de Ouagadougou, à savoir alliage Zone I (échantillons $\mathrm{n}^{\circ} 1$ ) et alliage Goughin I (échantillon $n^{\circ} 2$ ). Chaque alliage est unique. En fonction de leur composition chimique, les échantillons ont été dénommés comme suit : l'échantillon $\mathrm{n}^{\circ} 1$, qui contient de fortes teneurs en silicium et en cuivre combiné à un taux de magnésium faible et l'échantillon $n^{\circ} 2$ qui se différencie du précédent par de faibles teneurs en silicium et en cuivre avec un taux de magnésium élevé. Ces échantillons ont été comparés à de l'aluminium provenant d'un autocuiseur de fabrication européenne, appelé témoin. Les matières premières utilisées pour la fabrication des ustensiles de cuisine (Figure 1) proviennent d'une grande variété de déchets d'aluminium comme le montre la Figure 2. L'étude métallographique, les observations au microscope optique (MO), les analyses par Spectrométrie des photoélectrons $\mathrm{X}$ (XPS) et la diffraction de rayons X (DRX) nécessitent une bonne préparation de la surface. Ces alliages à analyser ont d'abord été polis mécaniquement à l'aide des papiers abrasifs en carbure de silicium par ordre de granulométrie décroissant (de 300 à 4000) et puis à la pâte diamantée (de $6 \mu \mathrm{m}$ à $1 \mu \mathrm{m}$ ) sur drap. Après ces deux séries de polissages, les échantillons ont été lavés à l'eau milli-Q, rincés successivement à l'acétone puis aux ultrasons dans de l'éther de pétrole et finalement séchés à l'air comprimé. Cette opération permet d'éliminer les incrustations ou impuretés encore présentes dans les échantillons.

\section{Attaque chimique}

Une attaque chimique avec le réactif de Flick (acide fluorhydrique 5\%) a permis d'observer les différentes phases présentes à la surface des échantillons (Figure 6). Ce réactif a pour rôle d'attaquer les limites des grains des alliages d'aluminium.

\section{Méthodes}

Afin de déterminer les éléments présents à la surface, des alliages d'aluminium ainsi que leur teneur, une analyse semiquantitative par fluorescence $X$ dans un premier temps a été appliquée. La DRX, l'XPS, la MO ont respectivement été utilisées pour la suite des analyses.

Analyse par Spectrométrie des photoélectrons X (XPS): Cette méthode permet une analyse et une identification des différents éléments présents à la surface des échantillons avant et après attaque en milieux culinaires. Le spectromètre utilisé est de type Physical Electronics PHI-5600. Au cours de l'acquisition, l'énergie de passage des électrons dans le détecteur (Pass Energy) a été fixée à 93,90 eV afin de maximiser le signal, pour la détection de traces. La calibration des spectres a été effectuée en fixant l'énergie de liaison du pic $\mathrm{C} 1 \mathrm{~s}$ de contamination à 284,6 eV. L'évaluation de l'environnement chimique ou état d'oxydation des éléments a été faite sur la base de valeurs reprises dans le livre de référence (Wagner et al., 1979).

Technique de spectrométrie fluorescence $\mathrm{X}(\mathrm{XRF})$ : Elle permet une analyse qualitative et semi quantitative en 
utilisant une propriété physique de la matière. Ainsi lorsque l'on bombarde de la matière avec des rayons $\mathrm{X}$, la matière réémet de l'énergie sous la forme, en autres, de rayons $\mathrm{X}$; c'est la fluorescence X (Ndiaye, 2006 ; Ramdé, 2009). Le spectre des rayons X émis par la matière est caractéristique de la composition chimique de l'échantillon, en analysant ce spectre, on peut en déduire la composition élémentaire. L'appareil utilisé pour la fluorescence est de type SRS 3000 Brüker.

Technique de diffraction de rayons $\mathrm{X}$ (DRX) : Cette méthode permet la mise en évidence de la nature des phases en présence. Le diffractomètre utilisé est de marque Brüker de type SIEMENS D5000 en mode $2 \theta$ et utilisant la radiation $\mathrm{K} \alpha$ du cuivre. Les spectres ont été enregistrés par pas de $\left(2 \Theta^{\circ}\right)=$ $0,02^{\circ}$, chaque pas durant 15 secondes, ce qui correspond à une vitesse de $0,08^{\circ} \cdot \mathrm{min}^{-1}$, et l'indexation a été effectuée entre 5 et $70^{\circ}$. (JCPDS X-ray Powder n ${ }^{\circ} 77-1715$; JCPDS Xray Powder n ${ }^{\circ} 87-2418$ )

Technique de Microscopie Optique (MO): La Microscopie Optique permet d'observer l'état des surfaces des échantillons d'aluminium fabriqués. Le microscope utilisé est de marque ZEISS JENAPOL équipé d'une caméra photographique qui permet l'obtention d'un grand nombre de photos des différentes microstructures à différents grossissements (Pike et al., 2002 ; INRS, 1999).

\section{Milieux d'études et de caractérisations}

Pour monter une opération similaire à la cuisine Burkinabé, nous avons placé les échantillons dans des béchers en pyrex contenant les différents milieux destinés à comprendre les phénomènes de corrosion :

$\mathrm{O}$ : eau de robinet $(300 \mathrm{ml})$ simple à température ambiante $; \mathrm{OS}_{1}$ : l'eau de robinet salée (titrée à $3 \mathrm{~g} / \mathrm{l}$ ou $9 \mathrm{~g} / \mathrm{l}$ ) à température ambiante ; $\mathrm{OS}_{2}$ : l'eau de robinet salée (titrée à $3 \mathrm{~g} / \mathrm{l}$ ou $9 \mathrm{~g} / \mathrm{l})$ à température d'ébullition; OR : riz (5 g dans $250 \mathrm{ml}$ d'eau de robinet); TC: concentré de tomate $(140 \mathrm{~g})$; $\mathrm{TF}$ : tomate fraîche ( $235 \mathrm{~g}$ pelée et transformée en purée). La cuisine se faisant la plupart du temps à chaud, les milieux $\left(\mathrm{OS}_{2}, \mathrm{OR}\right.$, TC et TF) sont testés à la température d'ébullition $\left(100{ }^{\circ} \mathrm{C}\right)$ pour simuler des conditions réelles de cuisson, température préconisée par la directive 97/48/CE (Altmann et al., 1999; Sarah, 2009).

\section{RÉSULTATS \\ Analyses chimiques des alliages}

Les résultats de l'analyse de spectrométrie à fluorescence $\mathrm{X}$ des échantillons sélectionnés ont été regroupés dans le Tableau 1, qui contient les résultats en pourcentage massique des éléments analysés en fonction de leur origine. On observe une grande variété de compositions chimiques des alliages qui résulte de l'extrême diversité de la qualité et de la quantité des matériaux composant le gisement des déchets d'aluminium. Le silicium, l'un des constituants majeurs des alliages d'aluminium destinés à la fonderie, permet d'abaisser la température de fusion des alliages, d'améliorer la coulabilité et par conséquent, de limiter les risques critiques (fentes dans le métal). La teneur en cuivre dans l'échantillon $\mathrm{n}^{\circ} 1$ est hors norme et les teneurs en zinc dans les deux échantillons sont supérieures à la norme. Mis à part, le chlorure et le manganèse, les autres éléments sont présents en faible quantité dans les deux échantillons. Tous les échantillons ont des teneurs en chlorure nettement supérieures à celles autorisées par la norme française, ceci étant probablement une conséquence de la charge de fusion dont il est issu. Les teneurs en fer et manganèse des alliages sont conformes à la norme. Ceci confirme ce que nous avons dit précédemment à savoir que, compte tenu de l'absence de règles prétablies pour la préparation de la charge de fusion et de la diversité des déchets d'aluminium, chaque alliage est unique. Le creuset en acier où l'aluminium en fusion qui demeure à une température élevée (plus de $650^{\circ} \mathrm{C}$ ) pendant 
plus d'une heure est aussi une source de contamination.

\section{Analyse par spectrométrie des photoélectrons X (XPS)}

La Figure 3 représente les spectres XPS globaux déterminés sur les échantillons $n^{\circ} 1$ et $\mathrm{n}^{\circ} 2$, après 60 minutes d'immersion dans les milieux d'études. Le spectre d'une surface polie est inclus à titre de comparaison. Le pic du carbone C 1s $(284,6 \mathrm{eV})$ a été utilisé comme référence en énergie. Onze (11) pics principaux ont été observés aux énergies de $1071,7 \mathrm{eV}, 932,5 \mathrm{eV}, 532 \mathrm{eV}, 399,5 \mathrm{eV}, 347,1$ $\mathrm{eV}, 284,6 \mathrm{eV}, 168,9 \mathrm{eV}, 133,2 \mathrm{eV}, 101,6 \mathrm{eV}$, $74,7 \quad \mathrm{eV}$ et $49,7 \mathrm{eV}$ correspondant respectivement au sodium, cuivre, oxygène, azote, calcium, carbone, soufre, phosphore, silicium, aluminium et magnésium. Après avoir déterminé les différents éléments présents à la surface des alliages d'aluminium, nous pouvons, à l'aide de ces spectres XPS à plus haute résolution, déterminer le degré d'oxydation de certains éléments en surface comme montré dans le Tableau 2. La présence de ces éléments sous différents états d'oxydation provient des milieux culinaires. En effet, il s'avère que les premières couches atomiques des différents échantillons sont composées d'oxyde d'aluminium $\left(\mathrm{Al}_{2} \mathrm{O}_{3}\right.$, Alumine) qui est dû probablement à leur oxydation avec l'air ambiant après nettoyage. Ces resultats sont en accord avec d'autres travaux de recherche.

\section{Analyse par diffraction des rayons $\mathrm{X}$ (DRX)}

Chaque famille d'alliage se caractérise par une valeur de d du plan (100). Les Figures 4 et 5 montrent les spectres des alliages étudiés en milieux culinaires et les pics présents correspondent à la structure c.f.c. de la matrice $\alpha$-aluminium. A l'exception du milieu OR pour lequel l'échantillon $n^{\circ} 2$ (Figure 5) présente un pic plus intense (311). Les diffractogrammes (milieux $\mathrm{OR}, \mathrm{OS}_{2}, \mathrm{TC}$, Figure 4) et (milieux $\mathrm{OS}_{2}, \mathrm{TC}$, Figure 5) présentent un pic plus intense (111), dont l'angle de diffraction est de $38,47^{\circ}$. Ceci suggère l'existence d'une orientation préférentielle ou texture. En effet, sur les différentes figures, l'intensité de la plus grande raie diminue en fonction du temps de cuisson et cette intensité reportée est une intensité relative. La diminution des grands angles observés atteste qu'il y a une variation du paramètre de réseau de la maille de la matrice $\alpha$-aluminium dû à la présence de certains éléments d'addition (Silicium, cuivre...). Ces résultats sont en bon accord avec d'autres travaux de recherche. Les spectres obtenus à partir de l'échantillon $\mathrm{n}^{\circ} 2$ dans les milieux $\mathrm{OS}_{2}$, OR et TC pendant 1 heure de cuisson apparaissent presque identiques à ceux obtenus dans le cas de l'échantillon $\mathrm{n}^{\circ} 1$ dans les mêmes milieux. Cependant, il y a apparition d'autres réflexions de la structure c.f.c. de la matrice $\alpha$ aluminium, due à une structure homogène c'est-à-dire une distribution relativement uniforme des éléments d'addition dans la matrice mère. Le dédoublement des pics (milieu TC pour l'échantillon $\mathrm{n}^{\circ} 1$ et milieu $\mathrm{OS}_{2}$ pour l'échantillon $\mathrm{n}^{\circ} 2$ ) est probablement dû à l'existence de deux longueurs d'ondes $\mathrm{K}_{\alpha 1}$ et $\mathrm{K}_{\alpha 2}$ du Cuivre qui sont très voisins. Cependant, sur la Figure 4 (milieux OR et $\mathrm{OS}_{2}$ ), de très petits pics sont présents : ce phénomène traduit la présence des traces de phases étrangères dans la matrice d'aluminium de l'échantillon $\mathrm{n}^{\circ} 1$ qui serait constituée de silicium, de cuivre, de la chalcopyrite $\left(\mathrm{CuFe}_{2}\right)$, du $\mathrm{Cu}_{3} \mathrm{Al}_{2}$, du $\mathrm{MnO}_{2}$ et du $\mathrm{Al}_{4,5} \mathrm{FeSi}$. L'existence de ces différentes phases dans cet alliage explique le décalage du pic intense vers la droite.

\section{Analyse par microscopie optique (MO)}

Une attaque chimique à l'acide fluorhydrique $5 \%$ permet l'observation des différentes phases. Un fond gris clair occupant 80 à $90 \%$ de la zone observée (Figure 6 b : Repère 1) : c'est la matrice d'aluminium; des zones de couleur noire très répandues dans les échantillons indiquent probablement la virulence de l'attaque de l'acide fluorhydrique 
(HF) (Figure 6 b : Repère 2); il s'agit des germes de silicium. Des lignes sombres sans contour définies semblent être issues des joints de grains; elles seraient composées d'un réseau de fines particules eutectiques dispersées dans la matrice d'aluminium (Figure 6 a: Repère 3). Les lignes sombres révèlent la présence de la phase silicium et jouent le rôle d'entaille dans la solution solide $\alpha$-Al ; les propriétés mécaniques de cette structure $\alpha$ sont faibles. En effet, la littérature affirme que près de l'alliage eutectique se trouvent le plus souvent des cristaux primaires de silicium, c'est-à-dire, qu'il y a accumulation de cristaux de silicium, qui ne sont pas fondus. Lors de la recristallisation, ils agissent comme germes cristallins du silicium, ce qui engendre la présence des zones de couleur noire dans les échantillons (Figure 6 $\mathrm{b}$ : Repère 2). De plus, la quantité de silicium présente dans les différents échantillons, conditionne dans une large mesure la microstructure des alliages étudiés. Au vue de ce qui précède, l'artisan doit procéder à un tri important des déchets riches en silicium en les regroupant par famille. Après l'observation des phases en milieu acide fluorhydrique $5 \%$, les attaques dans les différents milieux culinaires ont été observées comme suit :

$\mathrm{OS}_{1}$ : les échantillons $\mathrm{n}^{\circ} 1$ et $\mathrm{n}^{\circ} 2$ plongés à la température ambiante dans ce milieu (Figures 7 et 8) se dégradent au cours du temps. Au bout de 96 heures, quelques piqûres commencent à se manifester par attaque préférentielle de la matrice d'aluminium dans les deux échantillons et après 192 heures, les piqûres sont bien observables. L'échantillon $\mathrm{n}^{\circ} 1$ est plus attaqué, ceci étant probablement dû à la présence d'une grande quantité de silicium. Ces mêmes zones, observées avant et après le test, montrent une attaque du matériau au niveau des lignes de silicium et démontre que la matrice d'aluminium a subi une attaque préférentielle. Par colorimétrie, la présence d'ions aluminium a été détectée dans le milieu.
$\mathrm{OS}_{2}$ : l'échantillon $\mathrm{n}^{\circ} 1$ plongé dans ce milieu montre une rapide dégradation de la surface après une heure d'immersion, comme l'illustrent les Figures 9 et 10. Les mêmes zones, photographiées avant et après ce test, montrent une attaque du matériau au niveau des joints de grains (silicium) et des composés intermétalliques, laissant penser que la matrice d'aluminium a subi une attaque préférentielle. L'échantillon $n^{\circ} 2$ plongé dans ce même milieu montre, de façon encore plus évidente, une attaque du matériau dans la matrice d'aluminium autour des aiguilles de silicium Figure 11. De l'aluminium a encore été mis en évidence par colorimétrie dans la solution. L'expérience faite avec l'échantillon $\mathrm{n}^{\circ} 2$ dans les milieux $\mathrm{OR}, \mathrm{TC}$ et TF présente les mêmes caractéristiques (Figures 12, 13, 14). De l'aluminium a aussi été mis en évidence dans les solutions (milieux d'études) par colorimétrie. Dans les alliages d'aluminium, la microstructure est formée d'un mélange de phases, comprenant en majorité des cristaux d'aluminium primaire, des composés intermétalliques et du silicium. A l'issue de cette analyse, l'attaque des alliages issus du recyclage est gouvernée par la nature du milieu alimentaire (le milieu OR est le plus agressif), par la composition des alliages et par la structure micrographique. Par ailleurs, ce sont les solutions aqueuses qui sont à l'origine des dégradations de surface les plus importantes.

\section{Analyse chimique des substrats}

La colorimétrie nous a permis de mettre en évidence la présence d'ions $\mathrm{Al}^{3+}$ dans les substrats. Après avoir montré la possibilité de mise en solution des alliages d'aluminium par attaque au contact du milieu alimentaire, la recherche des produits de corrosion alimentaire dans l'aliment lui-même s'avère nécessaire. Les éléments recherchés sont l'aluminium, le cuivre, le zinc, le silicium, et quelquefois le plomb ou l'étain. Leur choix se justifie encore plus maintenant, à la suite de l'observation de leur composition chimique, de l'attaque essentiellement localisée dans la 
matrice d'aluminium et au niveau des interfaces de cette matrice avec les autres phases. Dans cette partie de l'étude, la cuisson a été prolongée jusqu'à 3 heures afin d'augmenter la concentration des ions issus de l'attaque dans les solutions. Les Tableaux 3,
4, 5 et 6 présentent, les résultats obtenus pour le milieu $\mathrm{O}, \mathrm{OS}_{2}$, OR et TC. Ces analyses montrent bien que les quantités d'éléments métalliques présentes dans les milieux d'attaques sont toutes très faibles dans l'intervalle des normes.

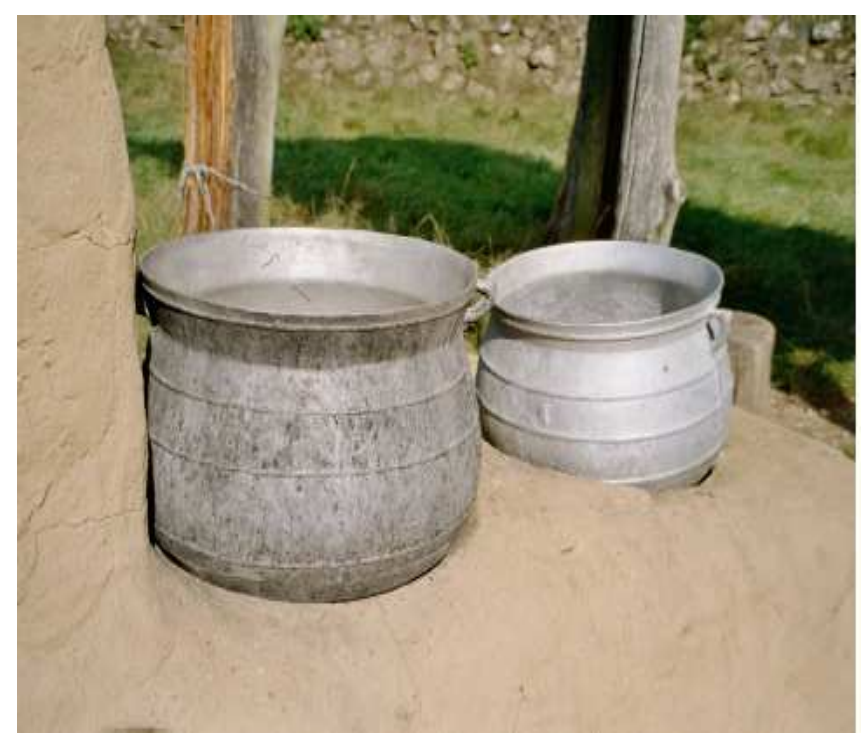

Figure 1 : Produits finis obtenus. Marmites artisanales de cuisine.
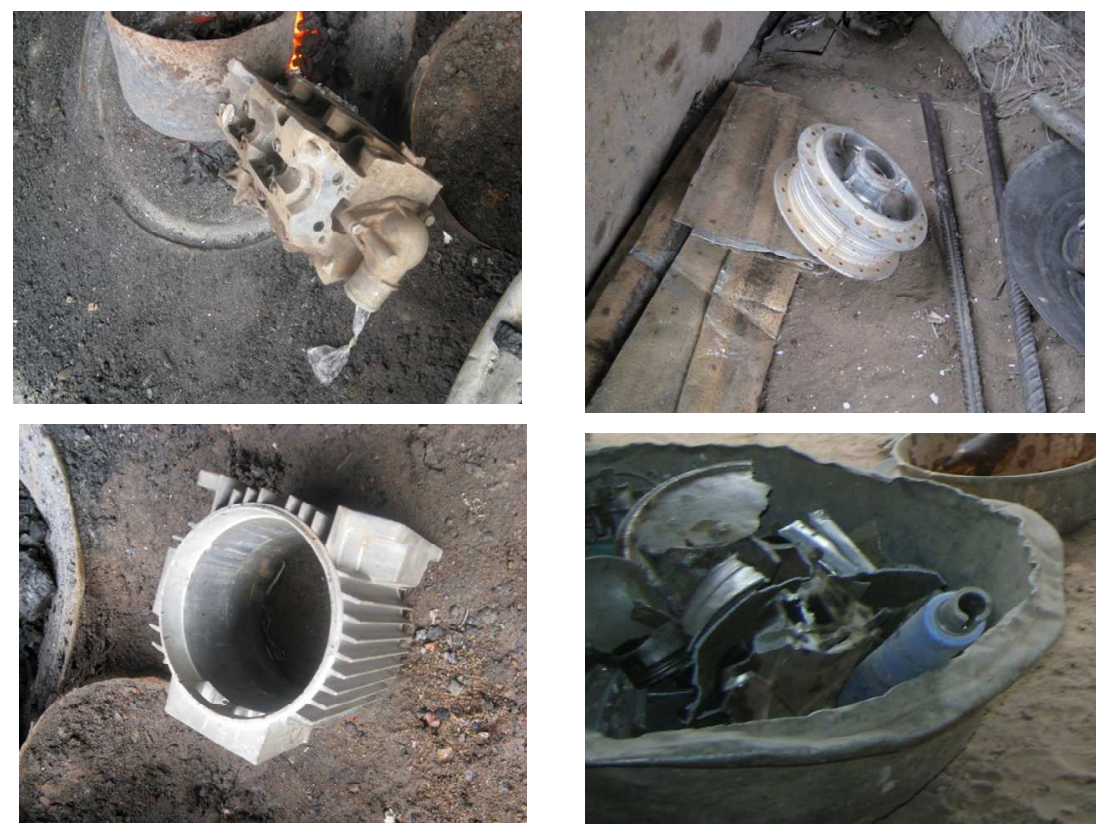

Figure 2 : Matières premières utilisées pour la fabrication des ustensiles de cuisines. 

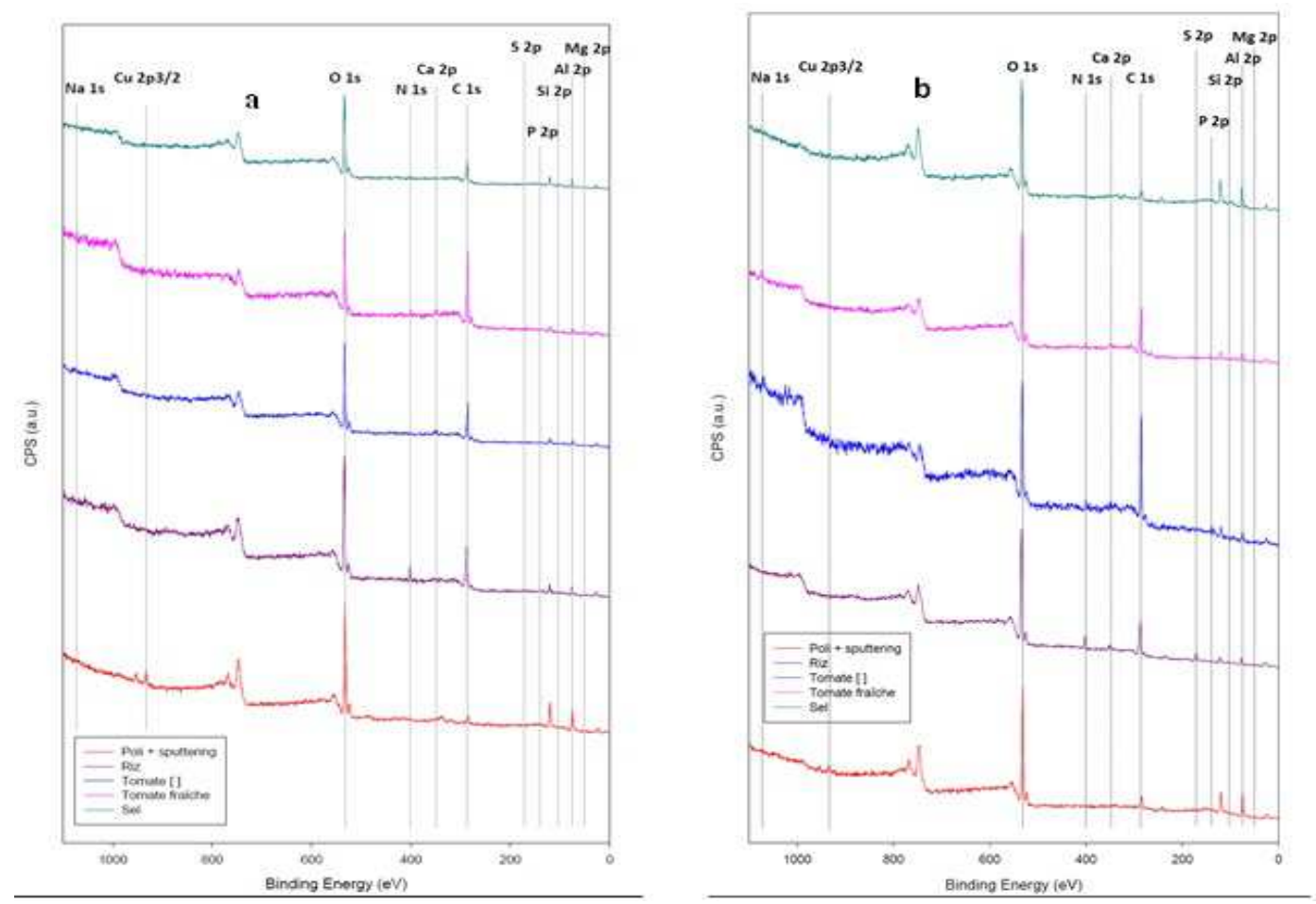

Figure 3 : Spectres XPS en surface des échantillons d'ustensile de cuisine dans les différents milieux culinaires. a) échantillon $n^{\circ} 1$, b) échantillon $n^{\circ} 2$.
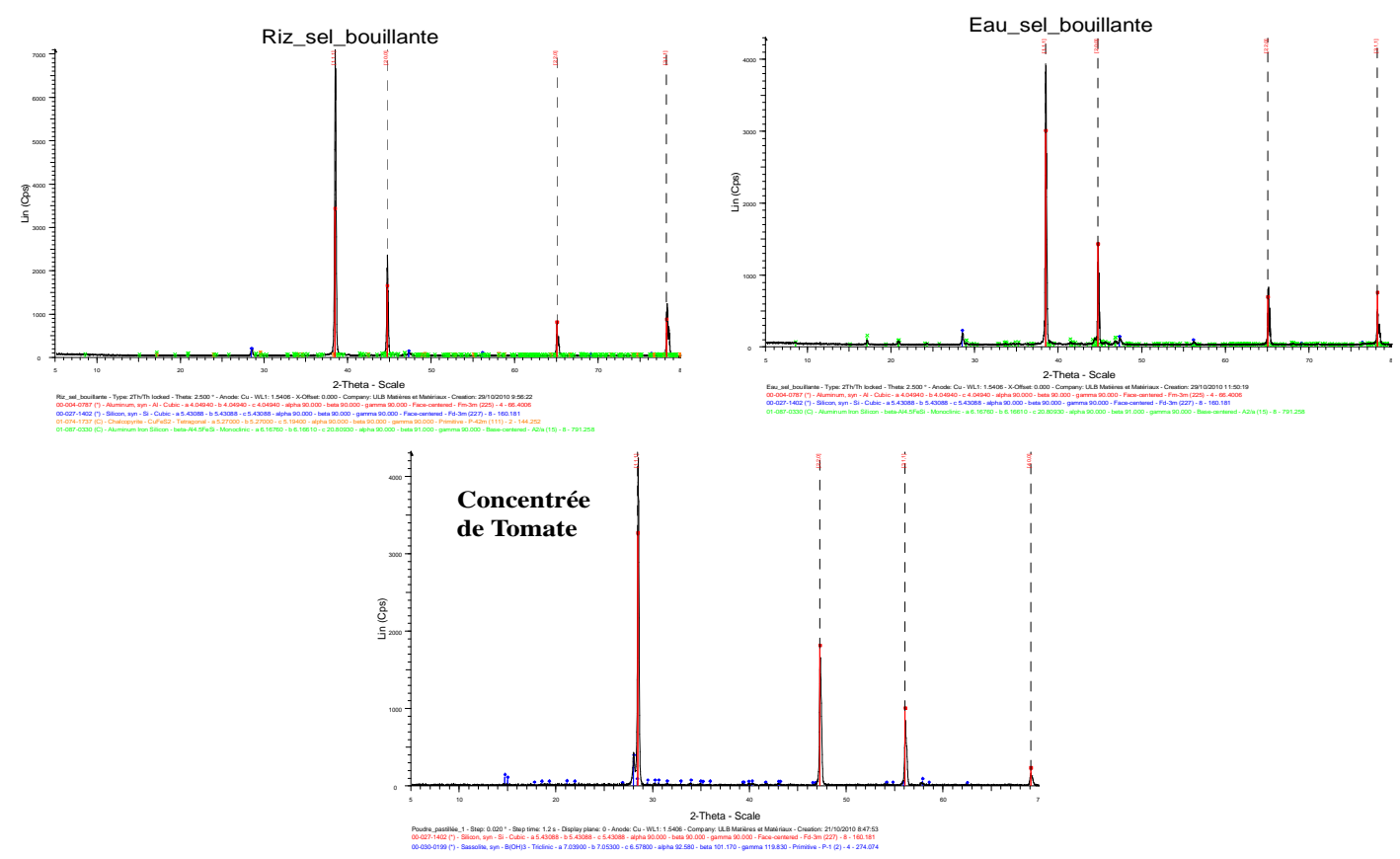

Figure 4 : Spectre de diffraction de rayons X (XRD) de l'échantillon $\mathrm{n}^{\circ} 1$ attaqué avec le sel, riz et tomate concentrée. 

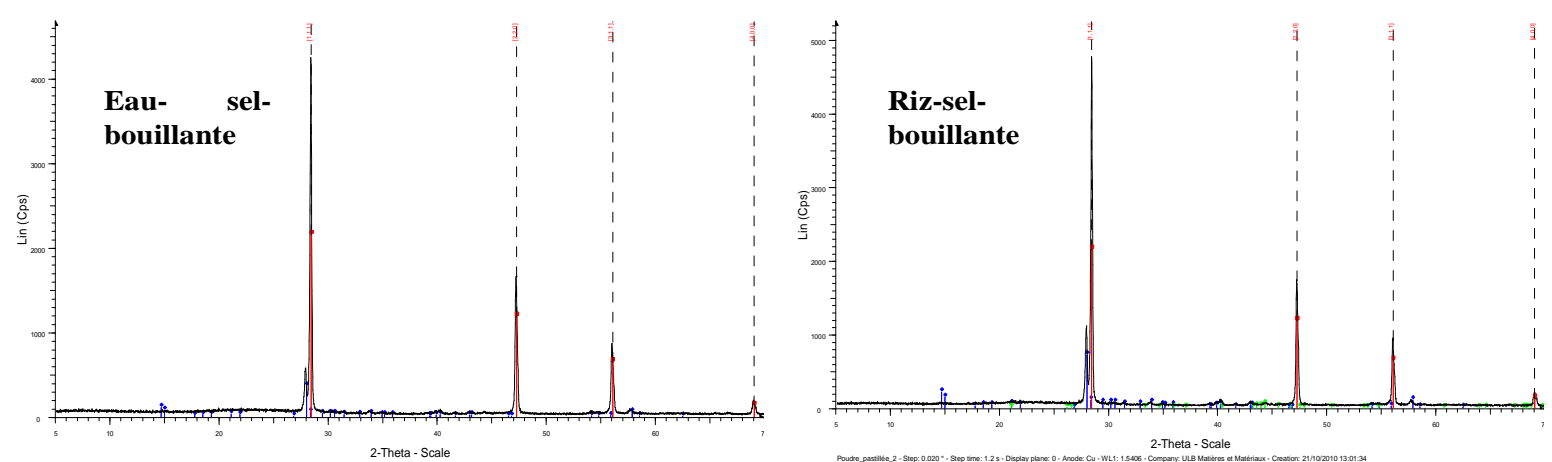

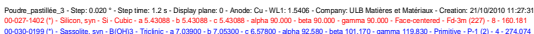
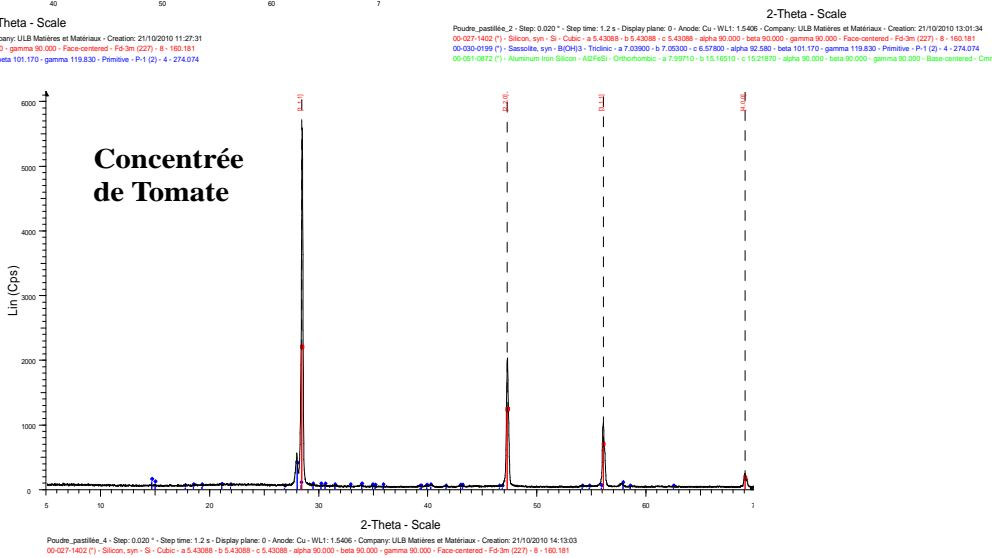

Figure 5 : Spectre de diffraction de rayons $X(X R D)$ de l'échantillon $n^{\circ} 2$ attaqué avec le sel, riz et tomate concentrée.
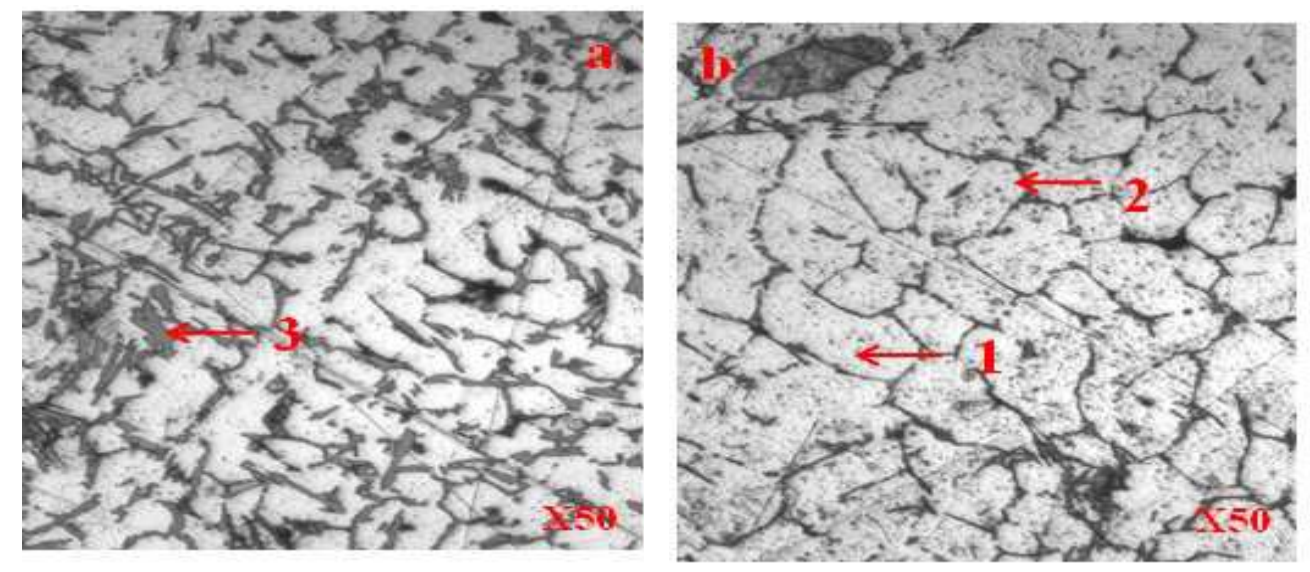

Figure 6: Images de la microscopie optique des alliages d'aluminiun du Burkina Faso après l'attaque chimique ( HF 5\%) illustrant les phases constituantes. a) Echantillon $n^{\circ} 1$ ( $12,7 \%$ de Silicium); b) Echantillon $n^{\circ} 2$ ( 4,27\% de Silicium). 


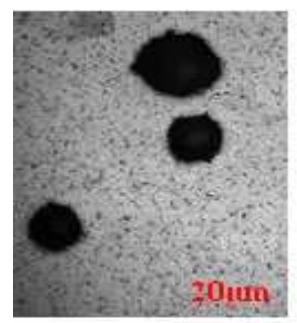

État initial

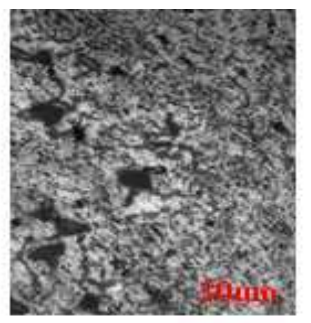

$3 \mathrm{~g} / 1$ (96h)

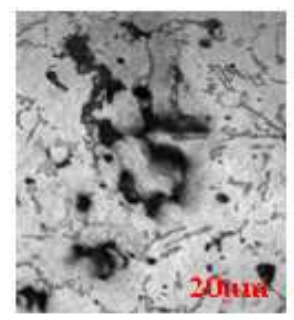

$9 \mathrm{~g} /(96 \mathrm{~h})$

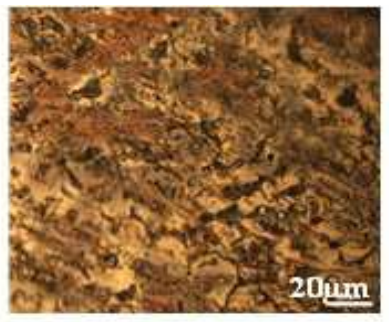

$9 \mathrm{~g} / 1(192 \mathrm{~h})$

Figure 7: Images micrographiques de la dégradation de l'échantillon $\mathrm{n}^{\circ} 1$ dans le milieu $\mathrm{OS}_{1}$ à la température ambiante.

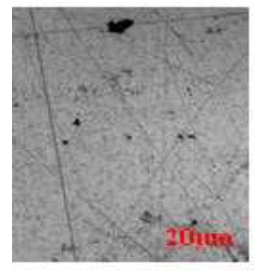

État initial

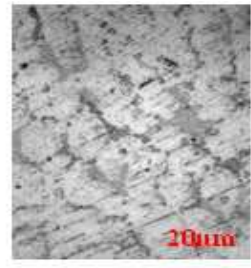

3g/1 (96h)

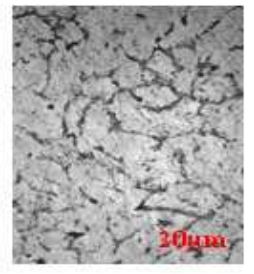

$9 \mathrm{~g} /(96 \mathrm{~h})$

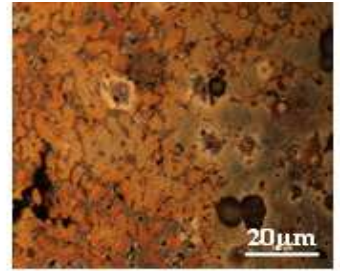

$9 \mathrm{~g} / 1$ (192 h)

Figure 8: Images micrographiques de la dégradation de l'échantillon $\mathrm{n}^{\circ} 2$ dans le milieu $\mathrm{OS}_{1}$ à la température ambiante.

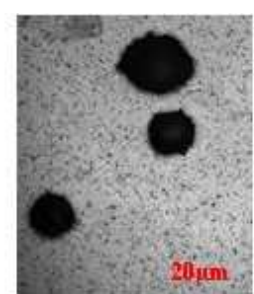

Etat initial

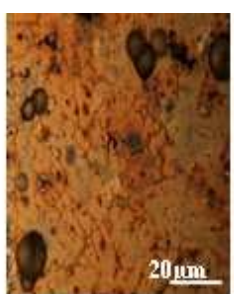

Après $60 \mathrm{mn}$

Figure 9: Échantillon $\mathrm{n}^{\circ} 1$ dans le milieu $\mathrm{OS}_{2}$ ( $\left.3 \mathrm{~g} / \mathrm{l}\right)$ pendant $60 \mathrm{mn}$.

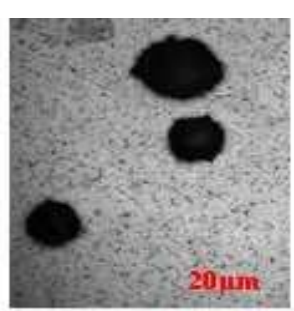

Etat initial

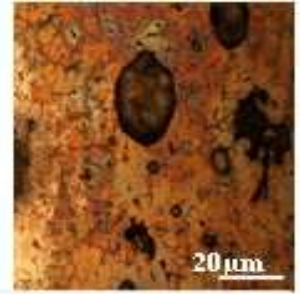

Après $60 \mathrm{mn}$

Figure 10: Échantillon $\mathrm{n}^{\circ} 1$ dans le milieu $\mathrm{OS}_{2}(9 \mathrm{~g} / \mathrm{l})$ pendant $60 \mathrm{mn}$. 


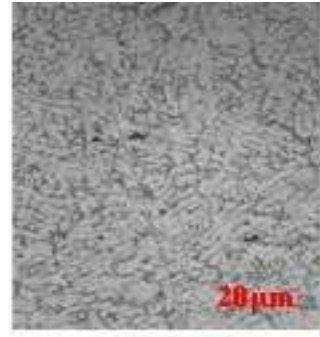

Etat initial

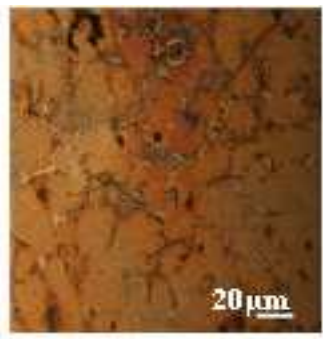

Ap rès $60 \mathrm{mn}$

Figure 11: Échantillon $\mathrm{n}^{\circ} 2$ dans le milieu $\mathrm{OS}_{2}(3 \mathrm{~g} / \mathrm{l})$ pendant $60 \mathrm{mn}$.

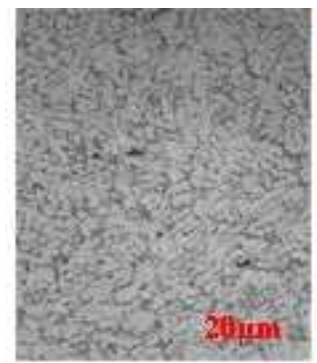

Etat initial

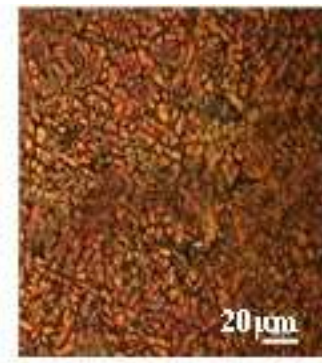

Ap rès $60 \mathrm{mn}$

Figure 12: Échantillon $\mathrm{n}^{\circ} 2$ dans le milieu OR porté à ébullition pendant $60 \mathrm{mn}$.

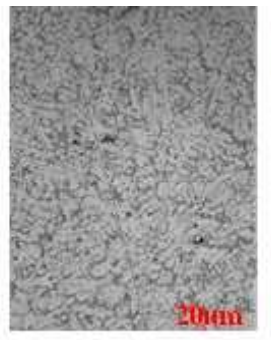

Etat initial

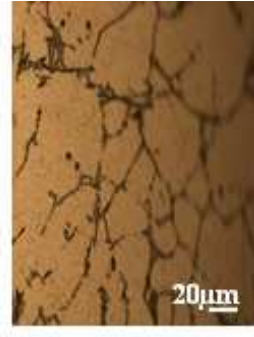

Après $60 \mathrm{~mm}$

Figure 13: Échantillon $\mathrm{n}^{\circ} 2$ dans le milieu TC porté à ébullition pendant $60 \mathrm{mn}$.

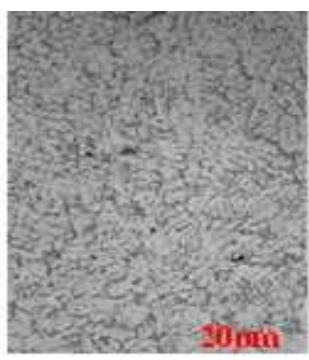

Etat initial

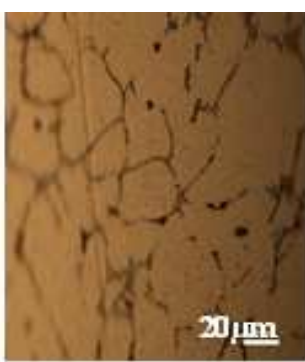

Apres $60 \mathrm{~mm}$

Figure 14: Échantillon $\mathrm{n}^{\circ} 2$ dans le milieu TF pelée porté à ébullition pendant $60 \mathrm{mn}$. 
Tableau 1 : Composition chimique (XRF) en \% massique des échantillons récoltés auprès des fondeurs du Burkina Faso et teneurs maximales admises par la norme NF EN 601 juillet 2004.

\begin{tabular}{|c|c|c|c|c|c|c|c|c|c|c|c|c|c|}
\hline Élements Échantillons & $\overline{A l}$ & $\mathbf{S i}$ & $\mathbf{F e}$ & $\mathbf{C u}$ & Zn & Mg & $\mathbf{C l}$ & $\mathrm{Cr}$ & Mn & $\mathbf{P b}$ & $\mathbf{N i}$ & $\mathbf{T i}$ & $\mathbf{Z r}$ \\
\hline \multirow[t]{2}{*}{ Échantillon 1} & $82,33 \pm$ & $12,70 \pm$ & $0,658 \pm$ & $1,11 \pm$ & $1,27 \pm$ & $0,24 \pm$ & $0,19 \pm$ & $0,021 \pm$ & $0,204 \pm$ & $0,0056 \pm$ & $0,31 \pm$ & $0,041 \pm$ & $0,012 \pm$ \\
\hline & 0,33 & 0,24 & 0,017 & 0,016 & $\mathbf{0 , 1 5 0}$ & 0,03 & $\mathbf{0 , 0 3}$ & 0,006 & 0,001 & 0,006 & 0,01 & 0,007 & $\mathbf{0 , 0 0 1}$ \\
\hline \multirow[t]{2}{*}{ Échantillon 2} & $93,24 \pm$ & $4,27 \pm$ & $0,601 \pm$ & $\mathbf{0 , 5 3 8 \pm}$ & $0,311 \pm$ & $0,48 \pm$ & $0,13 \pm$ & l & $0,055 \pm$ & $0,02 \pm$ & $0,045 \pm$ & $0,023 \pm$ & l \\
\hline & 0,34 & 0,15 & 0,016 & 0,011 & $\mathbf{0 , 0 1 0}$ & 0,03 & $\mathbf{0 , 0 3}$ & & 0,007 & 0,004 & 0,004 & 0,006 & \\
\hline \multirow[t]{2}{*}{ Témoin } & $96,84 \pm$ & $2,13 \pm$ & $0,19 \pm$ & $\mathrm{O}, 014 \pm$ & $0,018 \pm$ & $<0,01$ & $0,21 \pm$ & $<0,005$ & $<0,005$ & $<0,002$ & 0,011 & $<0,005$ & l \\
\hline & 0,34 & 0,11 & 0,010 & 0,003 & 0,002 & & $\mathbf{0 , 0 3}$ & & & & & & \\
\hline $\begin{array}{l}\text { Norme Française } 601 \text { de } \\
\text { juillet } 2004\end{array}$ & Reste & 13,5 & 2 & 0,6 & 0,25 & 11 & $<0,05$ & 0,35 & 4 & $<0,05$ & 3 & $\mathbf{0 , 3}$ & 0,3 \\
\hline
\end{tabular}

$\mathrm{Al}=$ Aluminium, $\mathrm{Si}=$ Silicium, $\mathrm{Fe}=\mathrm{Fer}, \mathrm{Cu}=$ Cuivre, $\mathrm{Zn}=$ Zinc, $\mathrm{Mg}=$ Magnésium, $\mathrm{Cl}$ = Chlorure, $\mathrm{Cr}=$ Chrome, $\mathrm{Mn}=$ Manganèse, $\mathrm{Pb}=\mathrm{Plomb}, \mathrm{Ni}=\mathrm{Nickel}, \mathrm{Ti}=\mathrm{Titane}, \mathrm{Zr}=\mathrm{Zirconium}$. 
Tableau 2: Composition chimique, nature des liaisons et du degré d'oxydation des éléments à la surface de l'aluminium.

\begin{tabular}{|c|c|c|}
\hline $\begin{array}{l}\text { Identification } \\
\text { des pics XPS }\end{array}$ & $\begin{array}{l}\text { Energie de } \\
\text { liaison }(e V)\end{array}$ & Commentaires/sources potentielle \\
\hline $\mathrm{Al} \mathrm{2p}$ & 74,7 & $\mathrm{Al}$ dans $\mathrm{Al}_{2} \mathrm{O}_{3}$ \\
\hline $\operatorname{Mg} 2 \mathrm{p}$ & 49,7 & Mg métallique \\
\hline Si $2 p$ & 101,6 & Si non métallique (oxyde de silicium) \\
\hline P $2 p$ & 133,2 & $\mathrm{P}$ dans des sels tels que $\mathrm{Na}_{2} \mathrm{HPO}_{4}$ ou $\mathrm{NaPO}_{3}$ \\
\hline S & 168,9 & $\mathrm{~S}$ sous forme de sulfates de métaux tels que $\mathrm{FeSO}_{4}$ ou $\mathrm{Fe}_{2}\left(\mathrm{SO}_{4}\right)_{3}$ \\
\hline $\mathrm{C} 1 \mathrm{~s}$ & 284,6 & Contamination de carbone, utilisée pour la calibration des spectres \\
\hline $\mathrm{Ca} 2 \mathrm{p}$ & 347,1 & Ca sous forme de $\mathrm{CaCO}_{3}$ ou d'halogénures de calcium $\left(\mathrm{CaX}_{2}\right)$ \\
\hline $\mathrm{N} 1 \mathrm{~s}$ & 399,5 & N sous forme d'amines (protéines, acides aminés...) \\
\hline $\mathrm{O} 1 \mathrm{~s}$ & 532,0 & Energie typique pour l'Oxygène sous forme $\mathrm{Al}_{2} \mathrm{O}_{3}: 532,2 \mathrm{eV}$ \\
\hline $\mathrm{Cu} 2 \mathrm{p}$ & 932,5 & $\mathrm{Cu}$ métallique ou $\mathrm{Cu}_{2} \mathrm{O}$ \\
\hline $\mathrm{Na} 1 \mathrm{~s}$ & 1071,7 & $\mathrm{Na}$ sous forme d'halogénures de sodium tel que le $\mathrm{NaCl}$ \\
\hline
\end{tabular}

Tableau 3 : Détection par colorimétrie d'ions dans le milieu $\mathrm{O}$ après 3 h de corrosion.

\begin{tabular}{lcccc}
\hline $\begin{array}{l}\text { Eléments } \\
\text { Échantillons }\end{array}$ & $\mathbf{A l}^{3+}(\mathbf{m g} / \mathbf{l})$ & $\mathrm{Cl}^{-}(\mathbf{m g} / \mathbf{l})$ & $\mathbf{F e}^{2+}(\mathbf{m g} / \mathbf{l})$ & $\mathbf{C u}^{2+}(\mathbf{m g} / \mathbf{l})$ \\
\hline Échantillon $\mathrm{n}^{\circ} 1$ & 5 & - & - & - \\
${\text { Échantillon } \mathrm{n}^{\circ} 2}^{\text {Témoin }}$ & 4 & - & - & - \\
\hline $\mathrm{Al}=$ Aluminium, $\mathrm{Cl}=$ Chlorure, $\mathrm{Fe}=\mathrm{Fer}, \mathrm{Cu}=$ Cuivre. & - & - & - \\
\hline
\end{tabular}

Tableau 4 : Détection par colorimétrie d'ions dans le milieu $\mathbf{O S}_{\mathbf{2}}$ après $3 \mathrm{~h}$ de corrosion.

\begin{tabular}{lcccc}
\hline $\begin{array}{l}\text { Eléments } \\
\text { Échantillons }\end{array}$ & $\mathbf{A l}^{3+}(\mathbf{m g} / \mathbf{l})$ & $\mathbf{C l}^{-}(\mathbf{m g} / \mathbf{l})$ & $\mathbf{F e}^{2+}(\mathbf{m g} / \mathbf{l})$ & $\mathbf{C u}^{2+}(\mathbf{m g} / \mathbf{l})$ \\
\hline${\text { Échantillon } \mathrm{n}^{\circ} 1}^{\circ}$ & 40 & 2300 & - & - \\
${\text { Échantillon } \mathrm{n}^{\circ} 2}_{\text {Témoin }}^{32}$ & 2300 & - & - \\
\hline
\end{tabular}

$\mathrm{Al}=$ Aluminium, $\mathrm{Cl}=$ Chlorure, $\mathrm{Fe}=\mathrm{Fer}, \mathrm{Cu}=$ Cuivre .

Tableau 5 : Détection par colorimétrie d'ions dans le milieu OR après $3 \mathrm{~h}$ de corrosion.

\begin{tabular}{lcccc}
\hline $\begin{array}{l}\text { Eléments } \\
\text { Échantillons }\end{array}$ & $\mathbf{A l}^{3+}(\mathbf{m g} / \mathbf{l})$ & $\mathbf{C l}^{-}(\mathbf{m g} / \mathbf{l})$ & $\mathbf{F e}^{2+}(\mathbf{m g} / \mathbf{l})$ & $\mathbf{C u}^{2+}(\mathbf{m g} / \mathbf{l})$ \\
\hline${\text { Échantillon } \mathrm{n}^{\circ} 1}^{\text {Échantillon }} \mathrm{n}^{\circ}$ & 50 & 2800 & - & - \\
Témoin & 40 & 2800 & - & - \\
\hline
\end{tabular}

$\mathrm{Al}=$ Aluminium, $\mathrm{Cl}=$ Chlorure, $\mathrm{Fe}=\mathrm{Fer}, \mathrm{Cu}=$ Cuivre. 
Tableau 6 : Détection par colorimétrie d'ions dans le milieu TC après $3 \mathrm{~h}$ de corrosion.

\begin{tabular}{lcccc}
\hline $\begin{array}{l}\text { Éléments } \\
\text { Échantillons }\end{array}$ & $\mathbf{A l}^{3+}(\mathbf{m g} / \mathbf{l})$ & $\mathbf{C l}^{-}(\mathbf{m g} / \mathbf{l})$ & $\mathbf{F e}^{2+}(\mathbf{m g} / \mathbf{l})$ & $\mathbf{C u}^{2+}(\mathbf{m g} / \mathbf{l})$ \\
\hline${\text { Échantillon } \mathrm{n}^{\circ} 1}$ & 50 & 3000 & - & - \\
Échantillon $\mathrm{n}^{\circ} 2$ & 40 & 3000 & - & - \\
Témoin & 60 & 3000 & - & - \\
\hline
\end{tabular}

$\mathrm{Al}=$ Aluminium, $\mathrm{Cl}=$ Chlorure, $\mathrm{Fe}=\mathrm{Fer}, \mathrm{Cu}=$ Cuivre .

\section{DISCUSSION}

Cette étude montre une plus faible résistance de l'échantillon $\mathrm{n}^{\circ} 1$ dans tous les milieux culinaires et ceci sera dû à sa plus forte teneur en silicium. Ces alliages doivent présenter une bonne coulabilité pour être mis en forme par fonderie avec des moules en sable (teneur en silicium assez élevée), mais aussi une bonne résistance à la corrosion pour leur comportement en milieu culinaire (teneurs en cuivre et fer faibles et bonne proportion de magnésium, les teneurs des autres éléments doivent être conformes aux normes). L'essentiel est de trouver un bon compromis pour des éléments comme le silicium dont une forte teneur garantit une bonne mise en forme en fonderie, mais une mauvaise résistance en corrosion. On privilégiera donc les alliages issus du milieu alimentaire (canette) et de celui de plaque d'imprimerie (Roberts et al., 1998) qui peuvent être mélangés avec d'autres, issus du milieu automobile (culasses, carters) ou de celui issu du bâtiment (cadre de fenêtre). Ces résultats sont en bon accord avec d'autres travaux de recherche (Caceres et al., 1999; Dinnis et al., 2006 ; Bartolomé et al., 2006 ). En effet, on peut remarquer les points suivants dans l'étude des échantillons récoltés au Burkina Faso:

$\checkmark$ La teneur en magnésium des alliages est fixée à $11 \%$ alors que la directive européenne ne mentionne pas cet élément ;

$\checkmark$ Le cuivre, le fer et le zinc sensibilisent les alliages à la corrosion intergranulaire. Leurs quantités admissibles dans les alliages sont faibles ; $\checkmark$ Le silicium ne fait pas l'objet de directives particulières quant à sa toxicité mais sa teneur est pourtant limitée à 13,5\%. Cette quantité correspond approximativement à la composition de l'alliage eutectique $\mathrm{Al} / \mathrm{Si}$, connu et utilisé pour sa bonne aptitude à la fonderie ;

$\checkmark$ Le plomb, comme nous l'avons vu est insoluble dans l'aluminium, mais pourtant, est limité à un niveau très bas suite à sa toxicité reconnue.

Pour un meilleur usage des déchets d'aluminium, afin d'améliorer l'utilisabilité alimentaire des alliages d'ustensiles de cuisine, qui est fortement liée à la composition chimique des alliages, les conclusions suivantes peuvent être tirées :

$\checkmark$ Les artisans doivent faire un tri judicieux, après la collecte pour sélectionner les déchets les plus aptes à entrer dans la confection des marmites artisanales ;

$\checkmark$ Les alliages d'ustensiles de cuisine (marmite artisanale) doivent présenter une bonne coulabilité pour être mis en forme par fonderie avec des moules en sable (alliages ayant des teneurs de silicium élevée) ;

$\checkmark$ Pour une bonne coulabilité, les alliages issus de matériaux destinés à l'usage alimentaire (canette...) doivent être privilégiés.

Les résultats montrent bien une présence importante d'ions aluminium dans les substrats. Cette présence pose le problème de la toxicité pour l'homme dans les substrats. En faisant l'hypothèse d'une perte de masse due à l'oxydation de l'aluminium dans l'alliage, dont la toxicité est sujette à controverses, qui est ensuite entièrement transféré à l'aliment, 
une personne consommant par exemple $250 \mathrm{~g}$ de ce riz ingérerait 9,8 mg de ce métal. A cette quantité d'aluminium provenant de la marmite s'ajoute celle apportée par les aliments. Par exemple, une eau potable contient environ 0,2 $\mathrm{mg} / \mathrm{l}$ d'aluminium (Belov et al., 2005 ; Abdelhak et al., 2014 ) et le riz en contient environ $1,7 \mu \mathrm{g} / \mathrm{g}$ (Herbulot, 2001 ; Wang et al., 2003). Les contributions de l'eau et du riz sont tellement faibles que la quantité d'aluminium ingérée est relativement indépendante de la proportion eau-riz. Or, les normes de toxicité retenues par certains auteurs (Xiang et al., 2006) font état d'une dose admissible journalière de $1 \mathrm{mg}$ par kilogramme de masse corporelle pour les humains. Cette dose est la quantité maximale tolérable par l'organisme humain au-delà de laquelle l'aluminium devient toxique pour lui (Woodson, 1998). Ce bilan simplifié montre que nous sommes loin du seuil critique pour lequel la santé humaine est mise en danger. D'après cette étude, nous pouvons conclure que les alliages rentrant dans la fabrication des marmites au Burkina Faso ne présentent pas de dangers particuliers sur le plan toxicologique.

\section{Conclusion}

Ce travail est une contribution à la caractérisation des ustensiles de cuisine dans les milieux culinaires, de deux (02) échantillons collectés dans deux (02) localités du centre du Burkina Faso (Ouagadougou). Les caractéristiques chimiques sont dépendantes de la provenance des échantillons d'ustensiles de cuisine. Ainsi les expériences que nous venons d'achever montrent que la dégradation est importante dans tous les milieux, en particulier dans ceux contenant du riz. Les données obtenues sont en outre conformes aux normes et montrent leur bonne qualité et leur usage possible pour la cuisson. La prédominance du silicium dans l'échantillon $\mathrm{n}^{\circ} 1$ est compatible avec l'agressivité dans les milieux étudiés et justifie leur forte attaque de cet échantillon. Cette étude a permis d'actualiser les données de la littérature et devrait soutenir l'intérêt agroalimentaire et socio-économique de la fabrication des ustensiles de cuisine dans le centre du Burkina Faso en fonction de la zone. A l'issue de quelques tests préliminaires, les alliages artisanaux du Burkina Faso sont inertes face aux aliments avec lesquels ils entrent en contact et nous remarquons des conséquences importantes sur la microstructure, plus particulièrement au niveau des interfaces entre l'aluminium et les autres phases. Ces matériaux sont donc bel et bien utilisables dans l'alimentation.

\section{REMERCIEMENTS}

Ce travail a été sponsorisé par la Coopération Universitaire pour le Développement (CUD)-Communauté française de Belgique. Nous tenons à remercier cette institution pour son soutien financier très important qui s'est traduit par des séjours de recherche de Jacques SAWADOGO à l'Université libre de Bruxelles, dans le cadre du projet de coopération institutionnelle entre la Communauté française de Belgique et l'Université de Ouagadougou.

\section{RÉFÉRENCES}

Abdelhak F, Debili MY, Lallouche S. 2014. Structural characterization of a bulk and nanostructured $\mathrm{Al}$ - Fe system, Materials and Technology, 48(5): 631-637.

Altmann P, Cunningham J, Dhanesha U, Ballard M, Thompson J, Marsh F. 1999. Disturbance of cerebral function in people exposed to drinking water contaminated with aluminium sulphate: retrospective study of the Camelford water incident. British Medical Journal, 319: 807-811.

Bartolomé M, Lopez V, Escudero E, Caruana G, Gonzalez J. 2006. Changes in the specific surface area of porous aluminium oxide films during sealing. Surface and Coatings Technology, 200: 4530-4537.

Belov NA, Eskin DG, Avxentieva NN. 2005. Constituent phase diagrams of the $\mathrm{Al}-\mathrm{Cu}-$ $\mathrm{Fe}-\mathrm{Mg}-\mathrm{Ni}-\mathrm{Si}$ system and their application 
to the analysis of aluminium piston alloys. Acta Materialia, 53: 4709 - 4722.

Caceres CH, Djurdjevic MB, Stockwell TJ, Sokolowski JH. 1999. The effect of $\mathrm{Cu}$ content on the level of microporosity in Al-Si-Cu-Mg casting alloys. Scripta Materialia, 40(5): 631-637.

Dinnis CM, Taylor JA, Dahle AK. 2006. Ironrelated porosity in $\mathrm{Al}-\mathrm{Si}-(\mathrm{Cu})$ foundry alloys. Materials Science and Engineering, A 425: 286 - 296.

Draissia M, Boudemagh H, Debili MY. 2004. Structure and Hardness of the Sputtered Al-Cu Thin Films System. Physica Scripta, 69: 348 - 350.

Draissia M, Debili MY, Millet JP. 2005. Comportement à la corrosion du système de films minces aluminium-cuivre. Journal of New Materials for Electrochemical Systems, 8: 229 - 233.

Draissia M, Debili MY. 2005. Study of solidsolution hardening in binary aluminiumbased alloys. Central European Journal Physical, 3 : 395 - 408.

Draissia M. 2004. Structure et physico-chimie des dépôts métastables aluminium-cuivre élaborés par pulvérisation cathodique magnétron. Thèse de doctorat, Université Badji-Mokhtar Annaba, Algérie, p. 98.

Herbulot F. 2001. Techniques de l'Ingénieur, Traité Matériaux métalliques, récupération et recyclage de l'aluminium, stratégie, dossier M 2345.

INRS. 1999. Valeurs limites d'exposition professionnelles aux agents chimiques en France. Cah Notes Doc Séc Trav., 174: 59-77.

Karbouj R. 2008. Transfert d'aluminium : Cas des matériaux pour contact alimentaire, Thèse de doctorat, Institut Polytechnique de Grenoble, Grenoble, P.67.

Ndiaye MB. 2006. Le recyclage de métaux d'origine industrielle au Sénégal, Thèse de doctorat, Ecole centrale de Lyon, Lyon, P. 85.
Pike LM, Anderson IM, Liu CT, Chang YA. 2002. Site occupancies, point defect concentrations, and solid solution hardening in $\mathrm{B} 2(\mathrm{Ni}, \mathrm{Fe}) \mathrm{Al}$. Acta Materials, 50(15) : 3859 - 3879.

Ramdé T. 2009. Comportement à la corrosion des alliages d'aluminium recyclés pour la confection de marmites, Thèse de doctorat, Université de Ouagadougou, Ouagadougou, p. 89.

Roberts NB, Clough A, Bellia JP, Kim JY. 1998. Increased absorption of aluminium from a normal dietary intake in dementia. Journal of Inorganic Biochemistry, 69: 171-176.

Sarah H. 2009. Etude du comportement en oxydation de nouveaux revêtements en aluminiure de Nickel dopé au Zirconiums : application aux barrières thermiques, Thèse de doctorat, Université pierre et Marie Curie, P.112.

Shabestari SG. 2004. The effect of iron and manganese on the formation of intermetallic compound in aluminum silicon alloys. Materials Science and Engineering, A 383: 289-298.

Wagner CD, Riggs WM, Davis LE, Moulder JF, Muilenberg GE. 1979. Handbook of $X-R$ ay Photoelectron Spectroscopy $\left(2^{\mathrm{e}}\right.$ edn). Perkin-Elmer: Eden Prairie.

Wang D, Shi Z, Zou L. 2003. Liquid aluminium corrosion resistance surface on steel substrat. Applied Surface Science, 214: 304-311.

Woodson GC. 1998. An interesting case of osteomalacia due to antacid use associated with stainable bone aluminium in a patient with normal renal function. Elsevier Science Inc., 22: 695-698.

Xiang C, Huiyuan G, Yanxiang L. 2006. Study on the eutectic modification level of Al-7Si Alloy by computer aided recognition of thermal analysis cooling curves. Materials Science and Engineering, A 419: 283-289. 